\title{
Precision medicine approaches in metabolic disorders and target organ damage: where are we now, and where are we going?
}

\author{
Amedeo Lonardo ${ }^{1}$, Christopher D. Byrne ${ }^{2,3}$, Giovanni Targher ${ }^{4}$ \\ ${ }^{1}$ Azienda Ospedaliero-Universitaria of Modena, Ospedale Civile di Baggiovara, Modena 41126, Italy. \\ ${ }^{2}$ Nutrition and Metabolism, Faculty of Medicine, University of Southampton, Southampton SO14, UK. \\ ${ }^{3}$ Southampton National Institute for Health Research Biomedical Research Centre, Southampton SO14, UK. \\ ${ }^{4}$ Endocrinology and Metabolism, Department of Medicine, University of Verona, Verona 37100, Italy.
}

Correspondence to: Prof. Amedeo Lonardo, Department of Internal Medicine, Ospedale Civile di Baggiovara, 1135 Via Giardini, Modena 41126, Italy. E-mail: a.lonardo@libero.it

\begin{abstract}
How to cite this article: Lonardo A, Byrne CD, Targher G. Precision medicine approaches in metabolic disorders and target organ damage: where are we now, and where are we going? Metab Target Organ Damage 2021;1:3.

https://dx.doi.org/10.20517/mtod.2021.03
\end{abstract}

Received: 9 Jun 2021 First Decision: 30 Jun 2021 Revised: 8 Jul 2021 Accepted: 20 Jul 2021 First online: 26 Jul 2021

Academic Editors: Ayako Suzuki, Saula Vigili de Kreutzenberg Copy Editor: Yue-Yue Zhang Production Editor: Yue-Yue Zhang

\begin{abstract}
In this review, we discuss selected topics which are relevant to implementing precision medicine in metabolic disorders. Personalization of diet and exercise may help in preventing obesity and type 2 diabetes (T2D). Weight loss should be personalized based on age, sex, ethnicity, and coexisting comorbidities. Advances in our understanding of the pathophysiology, genetics, and epigenetics of obesity promise to offer tailored management options. Careful risk assessment is necessary prior to intervention. Risk may be underestimated, e.g., in women, in different ethnic groups, and in people with T2D. More personalized approaches could be useful among persons who failed to respond to traditional risk factor management, such as pharmacological treatment for dyslipidemia and arterial hypertension. Nonalcoholic fatty liver disease/metabolic-associated fatty liver disease (NAFLD/MAFLD) is both a cause and an effect of altered glucose and lipid metabolism. Personalized medicine approaches could be key to identify more effective pharmacological strategies as well as to reverse this common and burdensome metabolic liver disease. Finally, metabolomics could be used to identify relevant biomarkers for cancer diagnosis, staging, and prognostication. Cancers of the colon and rectum, breast, prostate, thyroid, and ovaries illustrate the notion that cancer cell metabolic derangements may be utilized in clinical practice. A true personalization of pharmacotherapies should be pursued especially in obese patients with cancer.
\end{abstract}


Keywords: Arterial hypertension, biomarkers, breast, cancer, cardiovascular risk, epigenetics, genetics, gut microbiota, MAFLD, management, metabolic syndrome, metabolomics, NAFLD, pathophysiology, personalized medicine, precision medicine, prevention, prostate, research, thyroid, type 2 diabetes

\section{BACKGROUND}

\section{Definitions}

Metabolic disorders comprise a pathogenically and clinically heterogeneous set of conditions, which affect all age groups and exact a heavy clinical and financial burden ${ }^{[1]}$. Among the most prevalent metabolic disorders, obesity and type 2 diabetes (T2D) affect hundreds of millions of individuals globally and are projected to increase during the next decade ${ }^{[2]}$.

Precision medicine focuses on tailoring medical treatment to the individual characteristics of each patient and is aimed at improving clinical outcomes in a patient, thereby minimizing adverse events, while maximizing the therapeutic gains of any given treatment $t^{[3]}$. In precision medicine, the focus is on identifying which medical approaches will be effective for which patients based on genetic, environmental, and lifestyle factors ${ }^{[4]}$. There is a substantial overlap between the terms "precision medicine" and "personalized medicine". According to the National Research Council, the word "personalized" should not be used to imply that treatments and prevention are developed uniquely for each individual ${ }^{[4]}$. That said, the two terms are used synonymously and interchangeably in this article.

\section{Purpose}

Here, we try to identify some of the lines of research that promise to become relevant in the near future to implementing precision medicine in metabolic disorders. Rather than delivering a systematic overview for all metabolic diseases, we propose to focus on the metabolic syndrome (MetS) and its hepatic correlate, i.e., nonalcoholic fatty liver disease/metabolic-associated fatty liver disease (NAFLD/MAFLD). Additionally, we also address some cancer types that are strongly associated with MetS and its individual features. Finally, we also discuss the areas of potential development in this field.

\section{Methods}

We searched PubMed database using the following key words: "personalized medicine"; "precision medicine"; "metabolic syndrome"; "MAFLD"; "NAFLD"; "nonalcoholic steatohepatitis" (or "NASH”); "diagnosis and management"; and "cancer". Although this is a narrative rather than a systematic literature review, we have done our best to identify all pertinent articles published as of the date of our writing. Only those papers that were felt to be relevant based on the authors' agreement were retained.

\section{METABOLIC SYNDROME}

Prevention of obesity and T2D remains an unmet clinical and public health issue, and personalization of diet and exercise regimes is an important goal to help in achieving this aim. Pioneering observations between 1960 and 1989 from several researchers, including Denis Burkitt, eventually led to the so-called "fiber hypothesis" ${ }^{[5]}$. The dietary fiber hypothesis suggested that the risk of obesity, T2D, or heart attack increased as a result of fibers being removed during the refinement process of grains, rather than being due to a too high consumption of refined sugar. This had become commonplace in developed countries since 1760 , when the first industrial revolution took place in the $\mathrm{UK}^{[6]}$. Fibers reduce appetite, improve gut transit and microbiome composition, and, via microbial fermentation in the large bowel, increase the production of short-chain fatty acids (SCFA). SCFA, in turn, may induce epigenomic changes via metabolic regulatory receptors in distant organs, thereby promoting metabolic health through reducing the development of 
obesity, T2D, atherosclerosis, and certain cancer types ${ }^{[7]}$. How fibers are defined and what are the optimum amounts of daily fibers, which are necessary for maintaining and promoting metabolic health, is still poorly defined. However, it seems appropriate to define fiber as a complex mixture of dietary residues that convey health benefits, e.g., carbohydrate polymers (with three or more monomeric units), non-starch polysaccharides (from fruits and vegetables; cell wall components linked to polysaccharides), non-digestible oligosaccharides, and resistant starch, associated with non-carbohydrate substances (such as lignin) that are neither digested nor absorbed by the human small intestine but are metabolized by gut microbiota ${ }^{[7,8]}$.

Evidence suggests that an increased dietary fiber intake up to $50 \mathrm{~g} /$ day is associated with increased life expectancy, as well as improved quality of life added years and reduced healthcare expenditures ${ }^{[7]}$. Given the strong interactions of the intestinal microbiome with normal and diseased metabolism, the evaluation of inter-individual differences in microbiome composition and function promise to pave the way towards personalized microbiome-based medicine in treating common metabolic disorders belonging to the MetS $\operatorname{spectrum}^{[9]}$.

\section{Type 2 diabetes}

Awareness

Awareness of T2D risk among individuals with a positive family history for T2D may potentially improve risk-reducing behaviors. However, a recent study found that roughly one in four individuals were concordant between their perceived and clinical risk for $\mathrm{T} 2 \mathrm{D}^{[10]}$. This study suggested that personalized interventions should be carried out to specifically engage in early risk reduction strategies for those patients who tend to downplay and underestimate their overall T2D risk. Conceptually, similar findings have been identified for cardiovascular risk factors. Kim et al. ${ }^{[1]}$ conducted a cross-sectional survey of 1584 patients using a bilingual (English and Spanish) 32-item questionnaire, aimed at assessing understanding of cardiovascular risk factors. Data from this study show that, compared to White participants, AfroAmericans and Hispanics had a significantly higher misperception about their personal risk of T2D $(\mathrm{OR}=$ 2.22; 95\%CI: 1.08-5.57; and OR = 3.50; 95\%CI: 1.49-8.20, respectively) and Hispanics also for dyslipidemia $(\mathrm{OR}=2.21 ; 95 \% \mathrm{CI}: 1.19-4.10)$. This study strongly supports the notion that different ethnic groups have gaps between awareness and personalization of risk in major modifiable cardiovascular risk factors.

\section{Classification of diabetes subtypes}

Although invariably defined by chronic hyperglycemia, diabetes is characterized by a high level of clinical heterogeneity. Therefore, the identification of disease subtypes with different determinants at diagnosis and variable risk profiles remains a major aim of personalized medicine in the diabetes arena ${ }^{[12]}$. This could pave avenues for allocating resources especially to those patients who are at highest risk of developing chronic diabetic complications, as well as for enhancing power in experimental, genetic, and clinical studies by identifying more homogeneous patient populations ${ }^{[12]}$. With this backset, a consistent line of research has recently shown that a first step towards precision medicine in diabetes practice and research may be the stratification into homogenous subsets of patients. Recently, Ahlqvist et al. ${ }^{[13]}$ conducted a data-driven clustering of data from 8980 patients with newly diagnosed diabetes from the Swedish All New Diabetics in Scania cohort. Three large independent cohorts [the Scania Diabetes Registry $(n=1466)$, All New Diabetics in Uppsala $(n=844)$, and Diabetes Registry Vaasa $(n=3485)]$ were used for replication purposes, and clusters were based on six variables: glutamate decarboxylase antibodies, age at diagnosis, body mass index (BMI), HbA1c, and homoeostatic model assessment (HOMA) estimates of $\beta$-cell function and insulin resistance. Data show that five replicable clusters of patients with diabetes could be identified. Of these five different patient clusters, those in Cluster 3, which had more severe insulin resistance, were at the highest risk of diabetic kidney disease compared to those included in Clusters 4 and 5. However, treatment administered was similar across Clusters 3-5. Patients in Cluster 2 were more insulin deficient and had the 
highest risk of diabetic retinopathy ${ }^{[13]}$. Further evidence that distinct diabetes clusters may exhibit specific risk patterns of diabetes-related complications comes from the study by Zaharia et al. ${ }^{[14]}$. They performed a five-year follow-up study of a cohort of 1105 well-phenotyped patients with newly diagnosed type 1 or type 2 diabetes enrolled in the German Diabetes Study. At baseline, 35\% were classified as mild age-related diabetes (MARD), 29\% to mild obesity-related diabetes (MOD), $22 \%$ to severe autoimmune diabetes (SAID), $11 \%$ to severe insulin-resistant diabetes (SIRD), and $3 \%$ to severe insulin-deficient diabetes (SIDD). At five-year follow-up, 367 of these patients were re-assessed; 35\% of them had MARD, 29\% had MOD, 24\% had SAID, $10 \%$ had SIRD, and $3 \%$ had SIDD. In patients with newly diagnosed diabetes, intrahepatic fat content was highest at baseline in patients assigned to the SIRD cluster compared to all other clusters, irrespective of baseline glucose-lowering medications. Consistently, hepatic fibrosis at five-year follow-up was more pronounced in patients with SIRD than in those with SAID, MARD, MOD or SIDD. Confirmed diabetic sensorimotor polyneuropathy was also more prevalent at baseline in patients with SIDD than in those with SAID, MARD, MOD, and SIRD. Collectively, these findings suggest the conclusion that specific diabetes clusters have variably different risks of chronic diabetes complications.

\section{Management}

Achieving cardio-metabolic control is a largely unmet clinical goal that can best be obtained through personalization of drug treatment, in people with $\mathrm{T} 2 \mathrm{D}$. Conflicting with this practice gap, treatment trials are rarely designed to specifically address individual subsets of T2D patients (typically, elderly patients or those with chronic kidney injury) ${ }^{[15]}$. Therefore, additional studies should be aimed at suggesting optimized therapeutic strategies. A choice of drugs and dosages should be used to promote maximal benefit while keeping side effects to a minimum ${ }^{[15]}$. As discussed below, one approach useful to reaching this goal could be to move further to clinical phenotypes by using an omics-based approach. Initiatives from scientific societies are also expected to contribute to further promoting research in this area. For example, the Italian Association of Diabetologists (AMD) conducted an international survey to ascertain the opinions of physicians regarding those tailored therapeutic algorithms that have been developed by $\mathrm{AMD}^{[16]}$. Despite the inherent heterogeneity of $\mathrm{T} 2 \mathrm{D}$, the 452 participating physicians (the majority of whom were men and were diabetologists) widely agreed that there is a need for a personalized therapeutic approach that is accessible and easy to use $\mathrm{e}^{[16]}$.

Weight loss achievement and maintenance is a mainstay in T2D prevention and management. However, a recent study suggests that timing, extent, and BMI affect clinically relevant outcomes for weight loss maintenance, after a diagnosis of T2D. Strelitz et al. ${ }^{[17]}$ conducted an observational analysis on 2730 European individuals with screen-detected T2D. They reported that, compared to maintaining body weight, both weight loss and weight gain after screen-detected diagnosis of T2D were significantly associated with a higher risk of mortality, but not adverse cardiovascular events, particularly among non-obese individuals. This pioneering study suggests the importance of exploring avenues of personalization of weight loss advice. Additional parameters that predictably affected personalization of care in $\mathrm{T} 2 \mathrm{D}$ were age, sex, and ethnicity. Eiland et al. ${ }^{[18]}$, by reviewing the current state of diabetes technology adoption, found that "diabetes technology" (i.e., insulin delivery and glucose monitoring devices, as well as mobile medical applications and telemedicine) had modest effects in improving those disparities that result from age, sex, and even though this technology has great potential to improve disease complications and quality of life of people with T2D. However, the access to and use of technology is still very patchy. Additional research is needed to identify the specific grounds underlying disparities of access and use and how lacunas in health equity can be improved through targeted educational interventions while providing the "right" patient with the "right" technological tool. 
In the last years, accumulating evidence also supports the use a person-centered approach for treatment of type 2 diabetes, which is based on the individual risk of chronic vascular complications (i.e., CVD, CKD, or heart failure). Nowadays, the approach to diabetes therapy is very personalized and includes several combinations of antidiabetic agents with important favorable effects on CV risk, renal outcomes, and, of course, glucose management. In 2019, the new version of the ESC-EASD guidelines on "Diabetes, prediabetes and cardiovascular diseases" included the novel evidence generated over the last six years in large cardiovascular outcome trials with novel glucose-lowering drugs. The recommendations of these guidelines led to a completely novel positioning of medications for lowering blood glucose levels in the reduction of adverse cardiovascular and renal outcomes for patients with $\mathrm{T} 2 \mathrm{D}^{[19]}$.

\section{Obesity}

Obesity is a globally growing concern both in the general population and in specific patient groups. Given that various therapeutic approaches are now available ranging from lifestyle changes to drug options and endoscopic or surgical techniques, precision medicine is needed in this specific field of research to identify the best candidates and the best therapeutic approaches. For example, progresses in genetics and epigenetics of obesity might help in understanding the variable responses to treatments ${ }^{[20]}$.

Currently, dietary regimens remain a mainstay of treatment for the metabolic syndrome and its individual components, and personalization of diet is also a rational expectation for weight management and associated cardiovascular risk reduction. Recently, Ge et al. ${ }^{[21]}$ undertook a network meta-analysis of randomized trials to estimate the relative effectiveness of various types of diets, such as low carbohydrate (e.g., Atkins and Zone), low fat (e.g., Ornish), and moderate macronutrient (e.g., DASH and Mediterranean) diets, for weight and cardiovascular risk factor reduction in adults. Based on this metaanalysis of 121 randomized trials (21,942 participants), the authors showed that small to trivial differences exist among different dietary programs for weight and cardiovascular risk factor reduction, and these are often based on low certainty evidence. Of concern, weight reduction after 12 months of follow-up diminished in most of these randomized trials, and, aside from the Mediterranean diet for plasma LDL-C reduction, improvements in cardiovascular risk factors also largely disappeared. While the results of this meta-analysis could suggest prescribing a Mediterranean diet to specifically reduce hypercholesterolemia especially in obese individuals, further studies are necessary to better ascertain whether or not certain types of diets are more effective among specific obesity phenotypes.

Endoscopic placement of an intra-gastric fluid-filled balloon (IGB) is also a commonly used technique aimed at inducing weight loss, but this device is not devoid of side effects and requires removal in some patients. A pilot study conducted in 32 patients utilized a gastric emptying study, before and 2-3 months after placement of an IGB, to investigate intolerance and response to the single fluid-filled IGB ${ }^{[22]}$. These results show that utilization of baseline gastric emptying data could have prevented removal in $75 \%$ of cases. Moreover, decreased gastric emptying three months following IGB placement was associated with weight loss at 6 and 12 months. Although this study needs confirmation in a prospective validation cohort, it nicely proves the concept that the era of personalized endoscopic bariatric therapies has begun to help maximize the patients' tolerance, the cost-effectiveness ratio, and efficacy of intervention ${ }^{[22]}$.

Polygenic obesity in solid organ transplant (SOT) populations is deemed to be a risk factor for graft survival, owing to the development of metabolic disorders. However, little is known about the genetics of weight gain in SOT recipients. Saigi-Morgui et al. ${ }^{[23]}$ evaluated the association between weighted genetic risk scores and BMI, as well as the influence of clinical and genetic factors on $10 \%$ of weight gain, one-year after transplantation. They highlighted the importance of integrating the genetics of obesity in SOT recipients, 
which may contribute to treatment personalization and prediction of weight gain following SOT.

\section{Preventing cardiovascular disease in women}

Even though more women than men have died of cardiovascular disease (CVD) every year since $1984^{[24]}$, preventive recommendations have not been optimally applied to women ${ }^{[25]}$. In her article, Hayes ${ }^{[25]}$ highlighted that the emphasis of prevention has shifted toward assessment of a woman's "global" CVD risk as opposed to treatment of individual CVD risk factors. Interestingly, consolidated tools, such as the Framingham risk score, do take age, sex, smoking history, plasma lipids, and blood pressure into account ${ }^{[26]}$ and, may, therefore, be considered pioneering attempts to conduct a personalized medicine approach. Estimates of the overall CVD risk will dictate, in turn, tailored intervention intensity, with persons exposed to the most elevated CVD risk being the recipients of the most aggressive risk-lowering pharmacological strategies. This implies that, in the absence of any specific contraindications, women at a high CVD risk should be offered treatment with aspirin, beta-blockers, statins, and angiotensin-converting enzyme inhibitors or angiotensin receptor blockers whenever indicated. Furthermore, maintenance or even improvement of their healthy lifestyle practices should be encouraged for women who have already achieved a low CVD risk. Diligent application of preventive strategies is expected to effectively decrease CVD mortality and morbidity ${ }^{[26]}$.

\section{Hypertension}

In countries such as the Unites States, arterial hypertension (HTN) affects up to one third of adults. Current guidelines for the diagnosis and treatment of hypertension offer convenient heuristic algorithms for clinicians. However, they are limited by their involving little diagnostic testing and therefore are not highly personalized to the pathophysiology of individual patients, while more personalized treatment approaches might be useful ${ }^{[27]}$. Additionally, while a standardized treatment strategy for HTN will prove effective in most cases, a more personalized approach could be useful in selected patients, such as those who fail to respond to treatment ${ }^{[28]}$. The rationale for personalized therapy of HTN, obstacles to its implementation, together with examples of personalization measures, are discussed elsewhere ${ }^{[27]}$. A framework for the personalized treatment of HTN based, on the one hand, on a trade-off between simplicity and personalization and, on the other hand, on a classification of treatment approaches into low, medium, or high information burden approaches has also been proposed ${ }^{[28]}$.

\section{TARGET ORGAN DAMAGE}

\section{NAFLD/MAFLD}

NAFLD, which may be renamed and re-classified to MAFLD ${ }^{[29]}$, is a major challenge for contemporary hepatology, diabetology and internal medicine. This condition is the prototypic manifestation of a target organ (the liver) being associated, in a closed loop, with metabolic derangements, of which NAFLD is both a cause and an effect ${ }^{[30,31]}$. Confronted with the pathophysiological complexity and high clinical heterogeneity of this metabolic liver disease, attempts to conduct innovative treatment strategies have failed thus far ${ }^{[32]}$. Although many explanations may account for such failures, it seems logical to postulate that personalization of cure could be key to identifying more effective therapeutic approaches. Analysis of published papers on personalized medicine as applied to the NAFLD/NASH shows that many experts agree on identifying certain common grounds of interest in these areas: genetics/epigenetics, gut microbiota, and lysophospholipids/bile acids ${ }^{[33-37]}$.

The analytic discussion of all innumerable modifiers of pathogenic and clinical variables is beyond the scope of this article. Suffice it here to say that, in recent years, two main concepts have gained major interest: genetic (as opposed to metabolic) NAFLD and sexual differences in NAFLD arena. 
In their seminal paper, Luukkonen et al. ${ }^{[38]}$ profiled liver lipidome in 125 subjects with biopsy-proven NAFLD, who were classified into four groups based on their median HOMA-IR ("High and Low HOMAIR", $n=62$ and $n=63$ ) and PNPLA3 genotype [PNPLA3(148MM/MI), $n=61 v s$. PNPLA3(148II), $n=64$ ]. Data show that similar increases in steatosis extent and NASH prevalence were associated with a metabolically harmful saturated, ceramide-enriched liver lipidome in "metabolic NAFLD", but not in "genetic NAFLD", and this difference may account for why metabolic NAFLD (rather than genetic NAFLD owing to PNPLA3 polymorphism) increases cardiometabolic risk profile of these patients ${ }^{[38]}$. Together with other lines of evidence going in the same direction (reviewed $\mathrm{in}^{[3,40]}$ ), this study has conceptually paved the way towards renaming "metabolic NAFLD" to MAFLD.

Sex differences are a major modifier of health, diseases, medical practice, and research in many areas ${ }^{[41]}$. As regards NAFLD, a recent large meta-analysis of 54 observational studies (including a total of 62,239 individuals) showed that women had a $\sim 20 \%$ lower risk of NAFLD than men and, despite having a similar risk of NASH, they also had a higher risk of advanced fibrosis, especially after age 50 years ${ }^{[42]}$. These epidemiological data, together with the complex biological variations underlying sexual dimorphism in $\mathrm{NAFLD}^{[43]}$, strongly support the necessity to implement gender medicine for a personalized management of NAFLD.

In an attempt to dissect systematically, in the individual patient, the relative weight of the large gamut of NAFLD risk factors and clinical variables, Lonardo et al. ${ }^{[3,44,45]}$ recently proposed adoption of the so-called "LDE system". This includes a set of modifiers spanning from histologically/non-invasively defined liver histology (L) to determinants (D), such as sex/reproductive status, genetics, endocrine disorders, and (E) extra-hepatic manifestations of NAFLD (cardio-metabolic diseases and some cancers) that may address the unmet aim of achieving a personalized diagnosis. The LDE system or similar, logically structured, descriptors of the pathobiological complexity, promise to identify more homogenous subsets of NAFLD/MAFLD patients with comparable courses of disease and identifiable treatment responses.

\section{Cancer}

Cancer is a major global health problem issue, which exacts a heavy toll on healthcare systems ${ }^{[46]}$. Metabolomics defines a post-genomic research field, embracing small molecule analysis applied to utilizing biomarkers for cancer detection, monitoring, and prognostication ${ }^{[47]}$. Emerging discoveries of metabolomics promise to provide a suitable alternative for the current cancer diagnostic methods, notably including standard histopathological analysis ${ }^{[47]}$. Interestingly, formalin-fixed paraffin-embedded tissue specimens, which are commonly obtained for histopathological evaluation, are a valuable source for metabolomics investigations and can provide additional informative data for assessing disease stage and individual treatment responses. However, there remain some limitations to be addressed to optimize their utilization for metabolomics analysis ${ }^{[47]}$.

Precision oncology aims at selecting the best therapy for the "right" patient by considering variables related to both specific features of each individual's unique disease, as well as the patient's health status ${ }^{[48]}$. One of the biological phenomena underlying precision oncology is the fact that, in cancer, cells reprogram their metabolism to support all phases of disease including tumor initiation, progression, and metastasis, as well as drug resistance ${ }^{[49]}$.

Precision oncology can ideally be applied to patients with cancers of the colon and rectum, breast, prostate, thyroid, and ovaries, which are suitable paradigms for illustrating the notion that metabolic derangements of cancer cells may be targeted for therapeutic gain. Table 1 illustrates the concept of a personalized 
Table 1. A personalized approach based on metabolic analysis in some common types of cancer ${ }^{[48,50-53]}$

\begin{tabular}{|c|c|c|}
\hline $\begin{array}{l}\text { Cancer } \\
\text { types }\end{array}$ & Main findings & Ref. \\
\hline $\begin{array}{l}\text { Colorectal } \\
\text { (CRC) }\end{array}$ & $\begin{array}{l}\text { The following pathways are involved during CRC progression: redox status, energy metabolism, and intermediates of } \\
\text { amino acids, choline, and nucleotide metabolism }\end{array}$ & [50] \\
\hline Breast & $\begin{array}{l}\text { Nuclear magnetic resonance metabolomics may be used for phenotyping of breast cancer patients, using different bio- } \\
\text { specimens, such as tissues, blood serum/plasma, and urine }\end{array}$ & [48] \\
\hline Prostate & $\begin{array}{l}\text { Some urine metabolomics studies identified consistency in the dysregulation of } 15 \text { metabolites, while } 18 \text { metabolites } \\
\text { were found consistently altered in the prostate tissue, including alanine, arginine, uracil, glutamate, fumarate, and citrate } \\
\text { Of great interest are reports of altered valine, taurine, leucine, and citrate as the common denominators in both urine and } \\
\text { tissue studies, thereby emphasizing the human metabolome as a promising target for identifying novel biomarkers for } \\
\text { prostate cancer diagnosis }\end{array}$ & [51] \\
\hline Ovaries & $\begin{array}{l}\text { Downregulation of both phospholipids and histidine, citrulline, alanine, and methionine were the most often observed } \\
\text { biochemical changes. Compared to a single metabolite, the combination of multiple metabolites as a panel achieved a } \\
\text { better diagnostic accuracy }\end{array}$ & [52] \\
\hline Thyroid & $\begin{array}{l}\text { Identification of novel molecular markers of thyroid cancer promises to help identify distinct disease metabolic } \\
\text { phenotypes, which may lead to a more personalized therapy while assisting in both the diagnosis and prediction of } \\
\text { disease behavior }\end{array}$ & [53] \\
\hline
\end{tabular}

approach based on metabolic analysis in some common types of cancer.

\section{Colorectal cancer}

Globally, colorectal cancer (CRC) ranks among the principal causes of cancer-related mortality and is associated with Met ${ }^{[54,55]}$. The prognosis of CRC patients largely depends on prompt diagnosis and accurate staging of the disease. The latter can be achieved with novel non-invasive molecular tools, which characterize metabolite profiles associated with different cancer stages ${ }^{[50]}$. However, additional research is needed to reconcile the differences found among different studies in the levels of individual metabolites, which probably result from different study populations, sampling techniques, and data processing.

\section{Breast cancer}

Breast cancer is strongly associated with $\mathrm{Met}^{[56]}$. Given that breast cancer is an extremely heterogeneous disease, it is necessary to carefully stratify patients to maximize their survival rates while optimizing their quality of life. For example, the risk of recurrence and the potential benefits from adjuvant chemotherapies may be estimated with gene-expression tools ${ }^{[48]}$. However, additional research is needed to further reduce the proportion of breast cancer patients who are potentially exposed to unnecessary chemotherapy, and additional prognostic and stratification tools are eagerly awaited.

\section{Prostate cancer}

Globally, prostate cancer is the second most commonly diagnosed cancer in men, and a considerable body of evidence now suggests that abdominal obesity and chronic hyperinsulinemia are associated with a higher risk of this disease $\mathrm{e}^{[57,58]}$. One of the main limitations of using prostate-specific antigen serum levels is that they cannot invariably differentiate benign prostate hypertrophy from cancer, while identifying persons with indolent from those with aggressive prostate cancer ${ }^{[51,59]}$. Recent progresses in metabolomics, genomics, and proteomics could reveal new potential biomarkers ${ }^{[51]}$. In this connection, studies on metabolomics application for prostate cancer have focused on improved techniques to better analyze exosomes. These are extracellular vesicles which, being secreted from mammalian cells and virtually detected in all bio-fluids, are amenable to being used as tumor biomarkers ${ }^{[51]}$. Although data from pioneering clinical studies on potential prostate cancer biomarkers are still inconclusive ${ }^{[51]}$, this line of research will be better developed in the near future. In addition, metabolomics assessments in urine and prostate tissues are ideal approaches for disclosing specific changes that may occur with prostate cancer development ${ }^{[59]}$. 


\section{Ovarian cancer}

Multi-faceted and incompletely understood pathomechanisms explain the associations of obesity, T2D, or other metabolic derangements and increased risk of mortality due to ovarian cancer. These include dysregulated adipokine and cytokine profiles in subjects with excess adipose tissue and the ensuing altered immune responses and altered endocrine functions that can promote pro-tumorigenic signal transduction pathways ${ }^{[60]}$. Ovarian cancer is deemed to be "a silent killer", owing to the risk of late diagnosis resulting from non-specific clinical presentation and lack of any effective diagnostic techniques ${ }^{[61]}$. In this setting, in as much as they faithfully mirror tumor-host interactions, metabolomics profiles promise to be useful to improving ovarian cancer diagnosis, as well as identifying different metabolic signatures associated with different clinical phenotypes ${ }^{[01]}$. A recent systematic review evaluated metabolomics of biofluids and tumor tissue as potential biomarkers for the diagnosis, prognostication, and management response of ovarian cancer $^{[62]}$. Although findings addressing prognosis, treatment, and recurrence rates were more variable across various studies, lower levels of lysine mirrored either increased cell or glutamine dependency of rapidly proliferating cancer cells, suggesting that metabolic changes mirror cell proliferation and invasion associated with poor prognosis and higher recurrence rates ${ }^{[62]}$. To overcome those issues that limit their utility, further validation studies are awaited, aimed at introducing metabolites and biochemical pathways as significant aids in routine clinical practice.

\section{Thyroid cancer}

In general population studies, MetS is associated with an increased risk of incident thyroid cancer ${ }^{[63]}$. Thyroid cancer is the most common endocrine malignancy, and its diagnostic is fine needle aspiration biopsy (FNAB) ${ }^{[64]}$. However, FNAB is not exempt from indeterminate results and is often unable to differentiate different thyroid cancer types, thereby necessitating additional tissue sampling to achieve a definite diagnosis ${ }^{[6]}$.

Within this scenario, metabolomics studies have gained increasing popularity over the last decade [Table 1].

\section{Cancer in obese people}

Obesity is also a recognized risk factor for the development of various types of cancer ${ }^{[6]]}$. Additionally, obesity is associated with lower survival rates for many cancer types ${ }^{[67]}$. This close link between obesity and cancer development may be accounted for by different pathophysiological mechanisms which can promote the initiation and progression of tumors including chronic hyperinsulinemia, increased insulin-like growth factors, expanded and dysfunctional adipose tissue producing sex hormones, pro-inflammatory cytokines, and hypoxia ${ }^{[67]}$.

Although concerns of chemotherapy-related toxicity have been raised regarding obese cancer patients, some recent studies have shown that obese cancer patients do not experience more chemotherapy-related toxicity compared with their non-obese counterparts. This implies that what should be aimed at is a true personalization of therapy in obese patients with cancer rather than simple chemotherapy "depotentiation"[67]. In this connection, we know that achieving weight loss reduces overall cancer risk and improves compliance to therapy. This notion supports the implementation of social policies as well as medical/endoscopic/surgical therapies for obesity aimed at reducing cancer risk development, improving treatment outcomes and survival rates of people with obesity ${ }^{[67]}$.

\section{SARS-CoV-2 infection}

Starting from early 2020, the SARS-CoV-2 pandemic has exacted a major toll on global health, exerting a strong "stress test" on healthcare systems globally and enormously affecting world economics ${ }^{[6,69]}$. On these 
grounds, it comes as no surprise that research into this novel viral disease has revolutionized priorities in the research agenda ${ }^{[44]}$. Notably, a consistent line of research has identified a dangerous interaction of SARSCoV-2 infection with the host's metabolic health (as summarized in Table 2).

The data summarized in Table 2 suggest that perturbed metabolic homeostasis, such as occurring in people with obesity, T2D, or fibrosing MAFLD, may carry a greater risk of severe disease owing to SARS-CoV-2 infection. The mechanisms underlying such dangerous associations may be variably explained based on specific metabolic phenotypes. For example, not only do individuals with T2D have a low-grade inflammatory state, but they also may suffer from impaired leukocyte phagocytosis, neutrophil chemotaxis, bactericidal activity, and innate cell-mediated immunity. Collectively, these features predispose individuals with $\mathrm{T} 2 \mathrm{D}$ to more infectious disorders in general ${ }^{[70]}$. As specifically regards SARS-CoV-2 infection, people with $\mathrm{T} 2 \mathrm{D}$ may be prone to an increased risk of severe infection owing to an enhanced expression of the angiotensin-converting enzyme 2, which facilitates viral entry into cells. T2D might also facilitate the emergence of more pathogenic SARS-CoV-2 strains capable of causing greater severity of COVID-19 $9^{[70]}$.

As far as obesity is concerned, these individuals are also exposed to uncoordinated innate and adaptive immune responses, insufficient antibody response, and risk of SARS-CoV-2 infection-related cytokine "storm", i.e., a pro-inflammatory and pro-thrombotic state sustained by adipose tissue secreting cytokines and adipokines. Of concern, obesity could specifically worsen the severity of symptoms owing to COVID-19 among younger individuals ${ }^{[74]}$.

Finally, MAFLD/NAFLD with significant/advanced fibrosis may exacerbate the cytokine "storm" induced by SARS-CoV-2 infection, probably via the release of multiple proinflammatory hepatokines, thereby mechanistically contributing, in its turn, to the development of more severe illness owing to SARS-CoV-2 infection ${ }^{[72]}$.

\section{CONCLUSIONS AND RESEARCH AGENDA}

In this narrative review, we briefly discuss some key concepts that may be useful to implementing future research regarding personalized/precision medicine in the field pertaining to MetS, NAFLD/MAFLD, and cancer development. We analyze the evidence that prevention of obesity and T2D may be achieved with personalization of diet and exercise and other available management options. Women and all persons who are at the highest CVD risk should be offered the most complete and aggressive treatment options. NAFLD/MAFLD is mutually and bi-directionally associated with T2D and other metabolic disorders. Personalization of cure could be key to identifying more effective management approaches. Metabolomics could be effectively applied to utilizing biomarkers for cancer detection, monitoring, staging, and prognostication. Cancers of colon-rectum, breast, prostate, thyroid, and ovaries excellently illustrate the notion that (measurable) markers/biomarkers of cancer cell metabolic derangements can fruitfully be manipulated for clinical benefit. A true personalization of chemotherapy should be pursued in obese cancer patients. Weight loss reduces cancer risk and improves compliance to therapy. Very recent data suggest that dysregulated metabolic homeostasis, such as occurs in individuals with obesity, T2D, or fibrosing MAFLD, also increases the risk of severe disease owing to SARS-CoV-2 infection.

In this review, we present a view of research gaps in the field. Additional areas that, in our opinion, are worthy of future research include valuation of the impact of circadian clocks on normal metabolism and cardiometabolic disorders ${ }^{[75]}$; characterization of the hormonal profile, notably including thyroid profile, among persons with $\mathrm{MetS}^{[76]}$; and diagnosis and management of cardiovascular risk among persons with autoimmune or auto-inflammatory disorders ${ }^{[77-79]}$. 
Table 2. Evidence supporting a strong interaction between COVID-19 and metabolic disorders ${ }^{[70-73]}$

\begin{tabular}{|c|c|c|c|}
\hline Author, year & Method & Findings & Conclusion \\
\hline Targher et al. ${ }^{[70]}, 2020$ & $\begin{array}{l}\text { Retrospective study of } 339 \text { individuals } \\
\text { consecutively hospitalized at four sites in } \\
\text { China between January and February } 2020 \\
\text { COVID-19 was diagnosed with analysis of } \\
\text { oropharyngeal swab specimens } \\
\text { COVID-19 severity was classified as mild, } \\
\text { moderate, severe, or critical }\end{array}$ & $\begin{array}{l}\text { In the binary logistic regression analysis, the presence of T2D was associated with an } \\
\text { approximate } 4 \text {-fold increased risk of severe COVID-19 illness [odds ratio }(\mathrm{OR})=3.83,95 \% \mathrm{Cl} \text { : } \\
2.06-7.13, P<0.0001 \text { ] even after adjustment for confounding factors } \\
\text { Other strong predictors of severity of COVID-19 were older age, male sex, and obesity }\end{array}$ & $\begin{array}{l}\text { Having diabetes at hospital admission was } \\
\text { associated with an increased risk of severe } \\
\text { COVID-19 which was unaffected by } \\
\text { adjustment for confounders }\end{array}$ \\
\hline $\begin{array}{l}\text { Mantovani et al. }{ }^{[71]} \text {, } \\
2020\end{array}$ & $\begin{array}{l}\text { Meta-analysis of } 83 \text { studies involving } \\
78,874 \text { hospitalized patients with } \\
\text { laboratory-proven COVID-19 }\end{array}$ & $\begin{array}{l}\text { Pre-existent T2D was associated with an approximate } 2 \text {-fold higher risk of having } \\
\text { severe/critical COVID-19 illness (random-effects } \mathrm{OR}=2.10,95 \% \mathrm{Cl}: 1.71-2.57 ; 1^{2}=41.5 \% \text { ) and } \\
\sim 3 \text {-fold increased risk of in-hospital mortality (random-effects } \mathrm{OR}=2.68,95 \% \mathrm{Cl}: 2.09-3.44 ; \mathrm{I}^{2} \\
=46.7 \% \text { ). Funnel plots and Egger's tests did not reveal any significant publication bias }\end{array}$ & $\begin{array}{l}\text { Pre-existent diabetes, often type } 2 \text { diabetes, is } \\
\text { associated with a } 2-3 \text { times increased risk of } \\
\text { severe/critical illness and in-hospital mortality } \\
\text { owing to COVID-19 }\end{array}$ \\
\hline Targher, et al. ${ }^{[72]}, 2020$ & $\begin{array}{l}310 \text { consecutive patients with laboratory- } \\
\text { proven COVID-19 who had been } \\
\text { hospitalized at four sites in China, between } \\
\text { January and February } 2020 \\
\text { Hepatic steatosis was assessed with CT } \\
\text { scanning } \\
\text { The (low, intermediate, or high) risk of liver } \\
\text { fibrosis was assessed with FIB- } 4 \text { and NFS } \\
\text { COVID-19 was classified as severe and } \\
\text { non-severe }\end{array}$ & $\begin{array}{l}\text { The severity of COVID-19 markedly increased among MAFLD patients who had either } \\
\text { intermediate or high FIB-4 scores, and this association remained significant after adjusting for } \\
\text { sex, obesity and T2D } \\
\text { Similarly, the intermediate/high NFS (unadjusted-OR }=5.21,95 \% \mathrm{Cl}: 2.39-11.3 \text { ) was associated } \\
\text { with a higher risk of severe COVID-19 illness. This significant association persisted after } \\
\text { adjustments for sex, obesity, and T2D }\end{array}$ & $\begin{array}{l}\text { Patients with MAFLD with increased FIB-4 or } \\
\text { NFS scores are at higher risk of severe COVID- } \\
19 \text {, irrespective of metabolic comorbidities }\end{array}$ \\
\hline Gao et al. ${ }^{[73]}, 2020$ & $\begin{array}{l}\text { A cohort of } 150 \text { adults with COVID-19 from } \\
\text { three hospitals in China was enrolled } \\
75 \text { obese case subjects were randomly } \\
\text { matched with as many nonobese control } \\
\text { subjects paired by age and sex }\end{array}$ & $\begin{array}{l}\text { Obesity was associated with an approximately } 3 \text {-fold increased risk of having severe COVID- } \\
19 \text {, and a } 12 \% \text { increase in the risk of severe COVID-19 was observed per each 1-unit increase in } \\
\text { BMI } \\
\text { These associations remained significant even after adjusting for confounding factors }\end{array}$ & $\begin{array}{l}\text { Healthcare providers should be aware that } \\
\text { obese patients have an increased risk of } \\
\text { severe COVID-19 }\end{array}$ \\
\hline
\end{tabular}

BMI: Body mass index; COVID-19: coronavirus 19 disease; Cl: confidence interval; CT: computed tomography; FIB-4: Fibrosis 4; MAFLD: metabolic-associated fatty liver disease; NFS: NAFLD fibrosis score; OR: odds ratio.

\section{DECLARATIONS}

\section{Authors' contributions}

Made substantial contributions to conception and design of the study and performed data analysis and interpretation: Lonardo A 
Performed data acquisition, as well as provided administrative, technical, and material support: Byrne CD, Targher G

\section{Availability of data and material \\ Not applicable.}

\section{Financial support and sponsorship}

None.

\section{Conflicts of interest}

All authors declared that there are no conflicts of interest.

\section{Ethical approval and consent to participate \\ Not applicable.}

\section{Consent for publication}

Not applicable.

\section{Copyright}

(c) The Author(s) 2021.

\section{REFERENCES}

1. Lonardo A. Editorial: metabolism and target organ damage. Metab Target Organ Damage 2021;1:1. DOI

2. Kelly T, Yang W, Chen CS, Reynolds K, He J. Global burden of obesity in 2005 and projections to 2030. Int J Obes (Lond) 2008;32:1431-7. DOI PubMed

3. Lonardo A, Arab JP, Arrese M. Perspectives on precision medicine approaches to NAFLD diagnosis and management. Adv Ther 2021;38:2130-58. DOI PubMed PMC

4. Li, Rudolph E. Tanzi genetics of hidradenitis suppurativa. J Mol Genet 2020;3:1-7. DOI

5. Cummings JH, Engineer A. Denis Burkitt and the origins of the dietary fibre hypothesis. Nutr Res Rev 2018;31:1-15. DOI PubMed

6. Clark CG. Refined carbohydrate foods and disease. Some implications of dietary fibre. British Journal of Surgery 1976;63:898. DOI

7. O'keefe SJ. The association between dietary fibre deficiency and high-income lifestyle-associated diseases: Burkitt's hypothesis revisited. Lancet Gastroenterol Hepatol 2019;4:984-96. DOI PubMed PMC

8. Stephen AM, Champ MM, Cloran SJ, et al. Dietary fibre in Europe: current state of knowledge on definitions, sources, recommendations, intakes and relationships to health. Nutr Res Rev 2017;30:149-90. DOI PubMed

9. Shapiro H, Suez J, Elinav E. Personalized microbiome-based approaches to metabolic syndrome management and prevention. $J$ Diabetes 2017;9:226-36. DOI PubMed

10. Daack-Hirsch S, Shah LL, Jones K, et al. All things considered, my risk for diabetes is medium: a risk personalization process of familial risk for type 2 diabetes. Health Expect 2020;23:169-81. DOI PubMed PMC

11. Kim Y, Hogan K, D'Onofrio G, Chekijian S, Safdar B. Patient ethnicity predicts poor health access and gaps in perception of personal cardiovascular risk factors. Crit Pathw Cardiol 2017;16:147-57. DOI PubMed

12. Ahlqvist E, Prasad RB, Groop L. Subtypes of type 2 diabetes determined from clinical parameters. Diabetes 2020;69:2086-93. DOI PubMed

13. Ahlqvist E, Storm P, Käräjämäki A, et al. Novel subgroups of adult-onset diabetes and their association with outcomes: a data-driven cluster analysis of six variables. Lancet Diabetes Endocrinol 2018;6:361-9. DOI PubMed

14. Zaharia OP, Strassburger K, Strom A, et al. Risk of diabetes-associated diseases in subgroups of patients with recent-onset diabetes: a 5-year follow-up study. Lancet Diabetes Endocrinol 2019;7:684-94. DOI PubMed

15. Fodor A, Cozma A, Suharoschi R, Sitar-Taut A, Roman G. Clinical and genetic predictors of diabetes drug's response. Drug Metab Rev 2019;51:408-27. DOI PubMed

16. Gallo M, Mannucci E, De Cosmo S, et al. Algorithms for personalized therapy of type 2 diabetes: results of a web-based international survey. BMJ Open Diabetes Res Care 2015;3:e000109. DOI PubMed PMC

17. Strelitz J, Sharp SJ, Khunti K, et al. Association of weight loss and weight loss maintenance following diabetes diagnosis by screening and incidence of cardiovascular disease and all-cause mortality: an observational analysis of the ADDITION-Europe trial. Diabetes Obes Metab 2021;23:730-41. DOI PubMed

18. Eiland L, Thangavelu T, Drincic A. Has technology improved diabetes management in relation to age, gender, and ethnicity? Curr Diab Rep 2019;19:111. DOI PubMed

19. Cosentino F, Grant PJ, Aboyans V, et al; ESC Scientific Document Group. 2019 ESC guidelines on diabetes, pre-diabetes, and 
cardiovascular diseases developed in collaboration with the EASD. Eur Heart J 2020;41:255-323. DOI PubMed

20. Sun X, Li P, Yang X, Li W, Qiu X, Zhu S. From genetics and epigenetics to the future of precision treatment for obesity. Gastroenterol Rep (Oxf) 2017;5:266-70. DOI PubMed PMC

21. Ge L, Sadeghirad B, Ball GDC, et al. Comparison of dietary macronutrient patterns of 14 popular named dietary programmes for weight and cardiovascular risk factor reduction in adults: systematic review and network meta-analysis of randomised trials. $B M J$ 2020;369:m696. DOI PubMed PMC

22. Lopez-Nava G, Jaruvongvanich V, Storm AC, et al. Personalization of endoscopic bariatric and metabolic therapies based on physiology: a prospective feasibility study with a single fluid-filled intragastric balloon. Obes Surg 2020;30:3347-53. DOI PubMed

23. Saigi-Morgui N, Quteineh L, Bochud PY, et al; Swiss Transplant Cohort Study. Weighted genetic risk scores and prediction of weight gain in solid organ transplant populations. PLoS One 2016;11:e164443. DOI PubMed PMC

24. Statistical fact sheet 2013 update. Available from: https://www.heart.org/idc/groups/heartpublic/@wcm/@sop/@smd/documents/downloadable/ucm_319576.pdf [Last accessed on 26Jul 2021].

25. Hayes SN. Preventing cardiovascular disease in women. Am Fam Physician 2006;74:1331-40. PubMed

26. D'Agostino RB Sr, Vasan RS, Pencina MJ, et al. General cardiovascular risk profile for use in primary care: the Framingham Heart Study. Circulation 2008;117:743-53. DOI PubMed

27. Byrd JB. Personalized medicine and treatment approaches in hypertension: current perspectives. Integr Blood Press Control 2016;9:59-67. DOI PubMed PMC

28. Melville S, Byrd JB. Personalized medicine and the treatment of hypertension. Curr Hypertens Rep 2019;21:13. DOI PubMed PMC

29. Eslam M, Newsome PN, Sarin SK, et al. A new definition for metabolic dysfunction-associated fatty liver disease: an international expert consensus statement. J Hepatol 2020;73:202-9. DOI PubMed

30. Yki-järvinen H. Non-alcoholic fatty liver disease as a cause and a consequence of metabolic syndrome. Lancet Diabetes Endocrinol 2014;2:901-10. DOI PubMed

31. Lonardo A, Leoni S, Alswat KA, Fouad Y. History of nonalcoholic fatty liver disease. Int J Mol Sci 2020;21:5888. DOI PubMed PMC

32. Ratziu V, Friedman SL. Why do so many NASH trials fail? Gastroenterology 2020:S0016-5085(20)30680. DOI PubMed

33. Sharpton SR, Schnabl B, Knight R, Loomba R. Current Concepts, Opportunities, and challenges of gut microbiome-based personalized medicine in nonalcoholic fatty liver disease. Cell Metab 2021;33:21-32. DOI PubMed

34. Christinat N, Valsesia A, Masoodi M. Untargeted profiling of bile acids and lysophospholipids identifies the lipid signature associated with glycemic outcome in an obese non-diabetic clinical cohort. Biomolecules 2020;10:1049. DOI PubMed PMC

35. Cespiati A, Youngson NA, Tourna A, Valenti L. Genetics and epigenetics in the clinic: precision medicine in the management of fatty liver disease. Curr Pharm Des 2020;26:998-1009. DOI PubMed

36. Lorbek G, Urlep Ž, Rozman D. Pharmacogenomic and personalized approaches to tackle nonalcoholic fatty liver disease. Pharmacogenomics 2016;17:1273-88. DOI PubMed

37. Houghton D, Stewart CJ, Day CP, Trenell M. Gut microbiota and lifestyle interventions in NAFLD. Int J Mol Sci 2016;17:447. DOI PubMed PMC

38. Luukkonen PK, Zhou Y, Sädevirta S, et al. Hepatic ceramides dissociate steatosis and insulin resistance in patients with non-alcoholic fatty liver disease. J Hepatol 2016;64:1167-75. DOI PubMed

39. Wainwright P, Byrne CD. Bidirectional relationships and disconnects between NAFLD and features of the metabolic syndrome. Int $J$ Mol Sci 2016;17:367. DOI PubMed PMC

40. Lonardo A, Ballestri S, Targher G. "Not all forms of NAFLD were created equal". Do metabolic syndrome-related NAFLD and PNPLA3-related NAFLD exert a variable impact on the risk of early carotid atherosclerosis? Atherosclerosis 2017;257:253-5. DOI PubMed

41. Mauvais-jarvis F, Bairey Merz N, Barnes PJ, et al. Sex and gender: modifiers of health, disease, and medicine. Lancet 2020;396:56582. DOI PubMed PMC

42. Balakrishnan M, Patel P, Dunn-Valadez S, et al. Women have a lower risk of nonalcoholic fatty liver disease but a higher risk of progression vs men: a systematic review and meta-analysis. Clin Gastroenterol Hepatol 2021;19:61-71.e15. DOI PubMed

43. Lonardo A, Suzuki A. Sexual Dimorphism of NAFLD in Adults. Focus on clinical aspects and implications for practice and translational research. J Clin Med 2020;9:1278. DOI PubMed PMC

44. Lonardo A, Ballestri S. Perspectives of nonalcoholic fatty liver disease research: a personal point of view. Explor Med 2020;1:85-107. DOI

45. Lonardo A. Renaming NAFLD to MAFLD: Could the LDE system assist in this transition? J Clin Med 2021;10:492. DOI PubMed PMC

46. Huang A, Xie L. Healthinfo engineering. In: Management Association IR, editor. E-health and telemedicine. IGI Global; 2016. p. 53750. DOI

47. Tayanloo-Beik A, Sarvari M, Payab M, et al. OMICS insights into cancer histology; Metabolomics and proteomics approach. Clin Biochem 2020;84:13-20. DOI PubMed

48. Vignoli A, Risi E, McCartney A, et al. Precision oncology via NMR-based metabolomics: a review on breast cancer. Int J Mol Sci 2021;22:4687. DOI PubMed PMC

49. Liang L, Sun F, Wang H, Hu Z. Metabolomics, metabolic flux analysis and cancer pharmacology. Pharmacol Ther 2021;224:107827. DOI PubMed

50. Yusof HM, Ab-Rahim S, Suddin LS, Saman MSA, Mazlan M. Metabolomics profiling on different stages of colorectal cancer: a 
systematic review. Malays J Med Sci 2018;25:16-34. DOI PubMed PMC

51. Salciccia S, Capriotti AL, Laganà A, et al. Biomarkers in prostate cancer diagnosis: from current knowledge to the role of metabolomics and exosomes. Int J Mol Sci 2021;22:4367. DOI PubMed PMC

52. Ahmed-Salim Y, Galazis N, Bracewell-Milnes T, et al. The application of metabolomics in ovarian cancer management: a systematic review. Int J Gynecol Cancer 2021;31:754-74. DOI PubMed

53. Coelho M, Raposo L, Goodfellow BJ, Atzori L, Jones J, Manadas B. The potential of metabolomics in the diagnosis of thyroid cancer. Int J Mol Sci 2020;21:5272. DOI PubMed PMC

54. Rawla P, Sunkara T, Barsouk A. Epidemiology of colorectal cancer: incidence, mortality, survival, and risk factors. Prz Gastroenterol 2019;14:89-103. DOI PubMed PMC

55. Lee J, Lee KS, Kim H, et al. The relationship between metabolic syndrome and the incidence of colorectal cancer. Environ Health Prev Med 2020;25:6. DOI PubMed PMC

56. Wani B, Aziz SA, Ganaie MA, Mir MH. Metabolic syndrome and breast cancer risk. Indian J Med Paediatr Oncol 2017;38:434-9. DOI PubMed PMC

57. McGrowder DA, Jackson LA, Crawford TV. Prostate cancer and metabolic syndrome: is there a link? Asian Pac J Cancer Prev 2012;13:1-13. DOI PubMed

58. Daniyal M, Siddiqui ZA, Akram M, Asif HM, Sultana S, Khan A. Epidemiology, etiology, diagnosis and treatment of prostate cancer. Asian Pac J Cancer Prev 2014;15:9575-8. DOI PubMed

59. Lima AR, Pinto J, Amaro F, Bastos ML, Carvalho M, Guedes de Pinho P. Advances and perspectives in prostate cancer biomarker discovery in the last 5 years through tissue and urine metabolomics. Metabolites 2021;11:181. DOI PubMed PMC

60. Craig ER, Londoño AI, Norian LA, Arend RC. Metabolic risk factors and mechanisms of disease in epithelial ovarian cancer: a review. Gynecol Oncol 2016;143:674-83. DOI PubMed PMC

61. Saorin A, Di Gregorio E, Miolo G, Steffan A, Corona G. Emerging role of metabolomics in ovarian cancer diagnosis. Metabolites 2020;10:419. DOI PubMed PMC

62. Ahmed-Salim Y, Galazis N, Bracewell-Milnes T, et al. The application of metabolomics in ovarian cancer management: a systematic review. Int J Gynecol Cancer 2021;31:754-74. DOI PubMed

63. Park JH, Choi M, Kim JH, et al. Metabolic syndrome and the risk of thyroid cancer: a nationwide population-based cohort study. Thyroid 2020;30:1496-504. DOI PubMed

64. Huang LY, Lee YL, Chou P, Chiu WY, Chu D. Thyroid fine-needle aspiration biopsy and thyroid cancer diagnosis: a nationwide population-based study. PLoS One 2015;10:e127354. DOI PubMed PMC

65. Pinchot SN, Al-Wagih H, Schaefer S, Sippel R, Chen H. Accuracy of fine-needle aspiration biopsy for predicting neoplasm or carcinoma in thyroid nodules $4 \mathrm{~cm}$ or larger. Arch Surg 2009;144:649-55. DOI PubMed PMC

66. Pergola G, Silvestris F. Obesity as a major risk factor for cancer. J Obes 2013;2013:291546. DOI

67. Ottaiano A, De Divitiis C, Capozzi M, et al. Obesity and cancer: biological links and treatment implications. Curr Cancer Drug Targets 2018;18:231-8. DOI PubMed

68. Pivetta E. The COVID-19 pandemic: a stress test for clinical epidemiology. Epidemiol Prev 2020;44:28-9. DOI PubMed

69. Peter S. Goodman SARS stung the global economy. The coronavirus is a greater menace. The New York Times 2020.

70. Targher G, Mantovani A, Wang XB, et al. Patients with diabetes are at higher risk for severe illness from COVID-19. Diabetes Metab 2020;46:335-7. DOI PubMed PMC

71. Mantovani A, Byrne CD, Zheng MH, Targher G. Diabetes as a risk factor for greater COVID-19 severity and in-hospital death: a meta-analysis of observational studies. Nutr Metab Cardiovasc Dis 2020;30:1236-48. DOI PubMed PMC

72. Targher G, Mantovani A, Byrne CD, et al. Risk of severe illness from COVID-19 in patients with metabolic dysfunction-associated fatty liver disease and increased fibrosis scores. Gut 2020;69:1545-7. DOI PubMed

73. Gao F, Zheng KI, Wang XB, et al. Obesity is a risk factor for greater COVID-19 severity. Diabetes Care 2020;43:e72-4. DOI PubMed

74. Aghili SMM, Ebrahimpur M, Arjmand B, et al. Obesity in COVID-19 era, implications for mechanisms, comorbidities, and prognosis: a review and meta-analysis. Int J Obes (Lond) 2021;45:998-1016. DOI PubMed PMC

75. Sulli G, Manoogian ENC, Taub PR, Panda S. Training the circadian clock, clocking the drugs, and drugging the clock to prevent, manage, and treat chronic diseases. Trends Pharmacol Sci 2018;39:812-27. DOI PubMed PMC

76. Raposo L, Martins S, Ferreira D, Guimarães JT, Santos AC. Metabolic syndrome, thyroid function and autoimmunity - The PORMETS study. Endocr Metab Immune Disord Drug Targets 2019;19:75-83. DOI PubMed PMC

77. da Rosa Franchi Santos LF, Stadtlober NP, Costa Dall'Aqua LG, et al. Increased adhesion molecule levels in systemic lupus erythematosus: relationships with severity of illness, autoimmunity, metabolic syndrome and cortisol levels. Lupus 2018;27:380-8. DOI PubMed

78. Medina G, Vera-Lastra O, Peralta-Amaro AL, et al. Metabolic syndrome, autoimmunity and rheumatic diseases. Pharmacol Res 2018;133:277-88. DOI PubMed

79. Ebrahimi M, Ghayour-Mobarhan M, Rezaiean S, et al. Omega-3 fatty acid supplements improve the cardiovascular risk profile of subjects with metabolic syndrome, including markers of inflammation and auto-immunity. Acta Cardiol 2009;64:321-7. DOI PubMed 